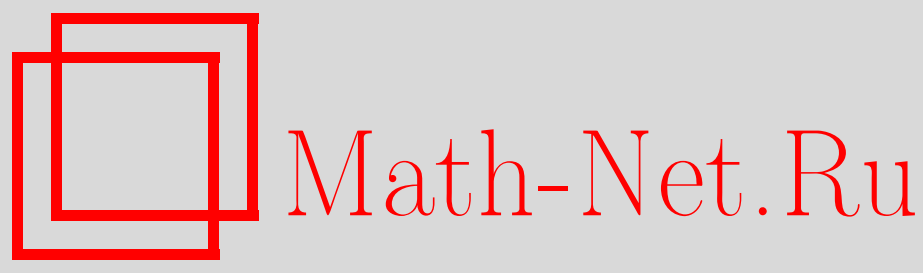

A. А. Нечаев, В. О. Попов, Обобщение теоремы Оре о неприводимых многочленах над конечным полем, Дискрет. матем., 2015, том 27, выпуск 1, 108-110

DOI: https://doi.org/10.4213/dm1318

Использование Общероссийского математического портала Math-Net.Ru подразумевает, что вы прочитали и согласны с пользовательским соглашением http://www . mathnet.ru/rus/agreement

Параметры загрузки:

IP : 52.90 .164 .192

26 апреля 2023 г., 16:07:43

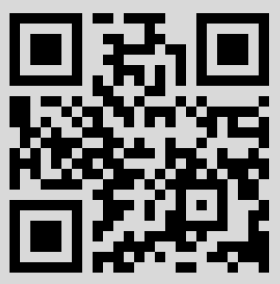




\section{Обобщение теоремы Оре о неприводимых многочленах над конечным полем}

() 2015 г. А. А. Нечаев* , B. О. Попов**

Для произвольного примарного числа $q$ приводится критерий неприводимости многочлена вида

$$
F(x)=x^{q^{m}-1}+a_{m-1} x^{q^{m-1}-1}+\ldots+a_{1} x^{q-1}+a_{0}, a_{0} \neq 0,
$$

над полем $K=G F\left(q^{t}\right)$.

Ключевые слова: неприводимые многочлены, критерий неприводимости.

Основной результат данной работы, анонсированный в [4], до сих пор не имел опубликованного доказательства. Здесь восполняется этот пробел.

Пусть $K=G F\left(q^{t}\right), q-$ степень простого числа $p$,

$$
F(x)=x^{q^{m}-1}+a_{m-1} x^{q^{m-1}-1}+\ldots+a_{1} x^{q-1}+a_{0}, a_{0} \neq 0,
$$

$S$ - минимальное поле разложение $F(x)$ над $K$ и $\sigma$ - автоморфизм Фробениуса поля $S$ над его подполем $P=G F(q): \sigma(a)=a^{q}$.

Рассмотрим кольцо $R=K[y ; \sigma]$ скрученных многочленов с обычным сложением и умножением, определяемым по дистрибутивности из соотношений

$$
y^{s} a=\sigma^{s}(a) y, a \in K, s \in \mathbb{N} .
$$

Напомним, что центр $Z(R)$ кольца $R$ состоит из всех многочленов вида $\sum_{i \geqslant 0} c_{i} y^{t i}$, $c_{i} \in P$.

Поставим в соответствие многочлену (1) многочлен

$$
f(y)=y^{m}+a_{m-1} y^{m-1}+\ldots+a_{0} \in R .
$$

В [1; стр. 82] показано, что в левом идеале $J=R f(y)$ содержатся ненулевые двусторонние идеалы и их сумма $I$ есть двусторонний идеал, называемый границей идеала $J$. Эта граница имеет вид $I=R f_{*}\left(y^{t}\right)$, где $f_{*}\left(y^{t}\right)-$ унитарный многочлен из $Z(R)$, который мы назовем границей многочлена $f(y)$. Многочлен $f_{*}\left(y^{t}\right)$ можно охарактеризовать также как унитарный многочлен наименьшей степени из $Z(R)$, который делится на $f(y)$ справа (напомним, что коэффициенты многочлена $f_{*}\left(y^{t}\right)$ принадлежат полю $P$ ). Основным результатом статьи является следующая теорема.

*Место работы: Академия криптографии Российской Федерации, e-mail: alexnechaev@inbox.ru

** Место работы: ООО "КРИПТО-ПРО" , e-mail: vpopov@cryptopro.ru 
Теорема 1. Многочлен (1) неприводим над полем $K=G F\left(q^{t}\right)$ тогда и только тогда, когда гранища $f_{*}\left(y^{t}\right)$ соответствующего ему многочлена (2) в кольще $R$ обладает свойствами

(a) $\operatorname{deg} f_{*}(x)=m$;

(б) $f_{*}(x)$ - многочлен максимального периода над полем $P=G F(q)$.

Заметим, что случай, когда $t=1$, исследован в [2]. В этом случае $K=P$, граница многочлена $f(y)$ совпадает с ним самим и неприводимость $F(x)$ равносильна тому, что $f_{*}(x)=f(x) \in P[x]$ есть многочлен максимального периода.

Доказательство. Из результатов, изложенных в [3; стр 26, 27], следует, что неприводимость многочлена $F(x)$ в $K[x]$ влечет за собой неприводимость многочлена $f(y)$ в $R$.

Лемма 1. Многочлен $f(y)$ неприводим в $R$ тогда и только тогда, когда

(a) $\operatorname{deg} f_{*}(x)=m$;

(б) $f_{*}(x)$ - неприводимый многочлен в $P[x]$.

Доказательство. Рассмотрим факторкольцо $\bar{R}=R / R f_{*}\left(y^{t}\right)$. Известно, что имеет место изоморфизм

$$
\bar{R} \cong M_{t}(A), \text { где } A=P[x] / f_{*}(x) .
$$

Очевидно, что многочлен $f(y)$ неприводим над $R$ тогда и только тогда, когда $R f(y)$ - максимальный левый идеал в $R$, а это, ввиду включения $R f_{*}\left(y^{t}\right) \subset R f(y)$, эквивалентно тому, что $\overline{R f}(y)$ - максимальный левый идеал в $\bar{R}$ (здесь $\bar{f}(y)$ - класс многочлена $f(y)$ в $\bar{R})$.

В [1; стр 80] доказано, что если $f(y)$ - неприводимый многочлен, то $R f_{*}\left(y^{t}\right)-$ максимальный двусторонний идеал в $R$, и потому $f_{*}(x)-$ неприводимый многочлен, т.е. кольцо $A$ из (3) есть поле. Если $\operatorname{deg} f_{*}(x)=k$, то $A=G F\left(q^{k}\right)$, и так как $\overline{R f}$ максимальный идеал в $\bar{R}=M_{t}(A)$, то $|\bar{R} / \overline{R f}|=q^{t k}$. С другой стороны, имеет место изоморфизм $R$-модулей $\bar{R} / \overline{R f} \cong R / R f$, и так как $\operatorname{deg} f(y)=m$, то $|\bar{R} / \bar{R} f|=|K|^{m}=q^{t m}$. Отсюда следует, что $k=m$, т. е. верны утверждения (а) и (б) леммы.

Обратно, пусть верны утверждения (а) и (б). Тогда $\bar{R}=M_{t}\left(G F\left(q^{m}\right)\right)$ ввиду (3), и так как $|\bar{R} / \overline{R f}|=q^{t m}$, то $\overline{R f}$ - максимальный левый идеал в $\bar{R}$, т. е. $f(y)-$ неприводимый многочлен в $R$. Лемма 1 доказана.

Продолжим доказательство теоремы 1. Пусть $\mathfrak{A}$ - алгебраическое замыкание поля $K$ и $\mathfrak{M}$ - множество корней многочлена $x F(x)$ в $\mathfrak{A}$. Нетрудно увидеть, что $\mathfrak{M}$ - векторное пространство над полем $P$ размерности $m$, инвариантное относительно линейного преобразования $\rho=\sigma^{t}$ (здесь $\sigma$ рассматривается как автоморфизм поля $\mathfrak{A}$, действующий по закону $\left.\sigma(\alpha)=\alpha^{q}\right)$. Ограничение $\tau$ преобразования $\rho$ на множестве $\mathfrak{M}$ есть циклический образующий группы Галуа многочлена $x F(x)$ над полем $K=G F\left(q^{t}\right)$. Зададим на $\mathfrak{A}$ операцию умножения "о" слева на элементы $R$ по правилу $h(y) \circ \alpha=h(\sigma)(\alpha)$. Тогда $\mathfrak{A}$ есть $R$-модуль и $\mathfrak{M}=\operatorname{Ker} f(\sigma)$. Поскольку многочлен $f(y)$ делит справа многочлен $f_{*}\left(y^{t}\right)$, то $\mathfrak{M} \subset \operatorname{Ker} f_{*}\left(\sigma^{t}\right)$. Значит, справедливо равенство

$$
f_{*}(\tau)=0
$$

Если $F(x)$ - неприводимый многочлен, то группа Галуа многочлена $x F(x)$ действует транзитивно на множестве $\mathfrak{M} \backslash\{0\}$ корней $F(x)$ и, значит, $\tau$ - цикл длины 
$q^{m}-1$, т. е. линейное преобразование пространства $\mathfrak{M}$ максимального периода. Ввиду леммы 1 в этом случае $f_{*}(x)$ - неприводимый многочлен степени $m$ и $f_{*}(x)-$ многочлен максимального периода в силу (4), то есть справедливы утверждения (а) и (б) теоремы.

Обратно, если выполнены условия (а) и (б), то из (4) следует, что $f_{*}(x)$ - характеристический многочлен преобразования $\tau$, где $\tau$ есть линейное преобразование пространства $\mathfrak{M}$ максимального периода. Следовательно, группа Галуа многочлена $F(x)$ над полем $K$ транзитивна на множестве его корней, т. е. этот многочлен неприводим над $K$.

Теперь видно, что для построения всех неприводимых над $K$ многочленов вида (1) нужно перебрать все унитарные многочлены максимального периода $f_{*}(x) \in P[x]$ степени $m$; их число равно $\frac{1}{m} \varphi\left(q^{m}-1\right)$. Затем для каждого такого $f_{*}(x)$ нужно перебрать все такие унитарные многочлены $f(y) \in R$ степени $m$, что $\overline{R f}$ - максимальный идеал в кольце $\bar{R}$, определяемом равенством (3). Количество таких многочленов $f(y)$ равно числу максимальных идеалов кольца $\bar{R}=M_{t}\left(G F\left(q^{m}\right)\right)$, т. е. равно $\frac{q^{m t}-1}{q^{m}-1}$. Отсюда следует последнее утверждение теоремы, поскольку по каждому построенному $f(y)$ однозначно строится соответствующий неприводимый $F(x) \in K[x]$ вида $(1)$.

\section{Список литературы}

1. Джекобсон Н., Теория колеи, М.: ГИИЛ, 1947, 266 с.

2. Ore O., "Contribution to the theory of finite fields", Trans. Amer. Math. Soc., 37:2 (1973), 241-242.

3. McDonald B. R., Finite Rings with Identity, New York: Markel Dekker, 1974.

4. Попов В. О., "Критерий неприводимости многочленов специального вида над конечным непростым полем", 5 Всесоюзный симпозиум по теории колец, алгебр и модулей. Тезисы сообщений, 1982, 104-105. 